\title{
Economic MPC with a contractive constraint for nonlinear systems
}

\author{
Defeng He, Jing Sun, Li Yu
}

\begin{abstract}
In this paper, we consider the stability issue of economic model predictive control (EMPC) for constrained nonlinear systems and propose a new contractive constraint formulation of nonlinear EMPC schemes. This formulation is one of Lyapunov-based approaches in which the contractive function chosen a priori can be used as a Lyapunov function. Some conditions are given to guarantee recursive feasibility and asymptotic stability of the EMPC. Moreover, we analyze the transient economic performance of the EMPC closed-loop system in some finite-time intervals. The proposed EMPC scheme is applied to a chemical reactor model to illustrate its utility and benefits.
\end{abstract}

Index Terms: Nonlinear predictive control, constrained control, economic optimization, stability, transient performance

\section{INTRODUCTION}

Economic model predictive control (EMPC) has received much attention because of its ability in integrating real-time process economic optimization and feedback control into an optimal control framework [1-2]. Unlike traditional MPC, where target-tracking controllers are computed by minimizing positive definite cost functions [3], EMPC directly utilizes general economic functions as stage costs to design controllers. Therefore, EMPC can directly address the operational requirements and hence, significantly improve system performance compared to the hierarchical control method in which an economically optimal operating point is computed by a real-time optimizer (RTO) in the upper layer and a target-tracking MPC in the lower layer is used to drive the system to the designed operating point $[1,4]$. However, in general EMPC cannot guarantee stability of operating points using traditional MPC techniques since it minimizes a general (not necessarily convex or positive definite) cost function over a finite prediction horizon [4].

A special way to address stability of EMPC is to establish economic criterion-based Lyapunov functions by modifying economic cost functions. For example, in [5] the economic cost function was transformed to the so-called rotated cost and then the monotonic decreasing property of the value function of the rotated cost is established by the assumption of strong duality and the terminal equality constraint. In [6], this terminal constraint was replaced by a terminal inequality constraint and a terminal penalty. Moreover, the assumption of strong duality was relaxed as a dissipativity condition with some elaborately chosen supply and storage functions, and the closed-loop stability of the optimal steady state was ensued by the dissipativity condition [4,7-8]. In [9] a generalized terminal equality constraint and a weighted terminal penalty were presented for both EMPC and target-tracking MPC, however with no stability analysis. The authors in [10-11] presented a stabilizing EMPC without terminal constraints by imposing controllability conditions and using a sufficiently long prediction horizon. However, a long horizon makes the EMPC optimization problem a challenge to solve, thereby causing difficulties in real-time applications.

Another way to guarantee stability of EMPC is to enforce Lyapunov-based stability constraints to optimization problems of EMPC. For instance, [12] proposed a Lyapunov-based EMPC scheme which uses two different modes of operation such that the closed-loop system is ultimately bounded in a small region. In [13], the Lyapunov-based EMPC [12] was modified as a double-layer stabilizing EMPC structure, where three (economic) MPC controllers with different prediction horizons were considered and stability of the closed-loop system was guaranteed by a controllability assumption. As one case of lexicographic multiobjective MPC [14], lately [15] proposed a Lyapunov-based EMPC scheme in a double-layer framework in which economic performance and stability were viewed as two conflicting objectives. To make a tradeoff for both objectives, a contractive constraint was designed using an upper-layer tracking-target MPC subject to a terminal equality constraint. Then this constraint imposed to the lower-layer economic MPC was employed to ensure stability of the EMPC, together with inherent robustness [16] of the upper-layer MPC. Besides solving two constrained optimization problems at each time, the global optimality of the upper-layer MPC is not necessarily guaranteed because of the non-convex nature of the

This work is supported by the National Natural Science Foundation of China under Grant 61374111 and the Public Welfare Technology Application Research Project of Zhejiang Province under Grant 2015C31057.

D.F. He and L. Yu are with the College of Information Engineering, Zhejiang University of Technology, Hangzhou, Zhejiang 310023 P.R. China. D.F $\mathrm{He}$ is also with the Department of Naval Architecture and Marine Engineering, University of Michigan, Ann Arbor, MI 48109 USA (e-mail: hdfzj@zjut.edu.cn; lyu@zjut.edu.cn).

J. Sun is with the Department of Naval Architecture and Marine Engineering, University of Michigan, Ann Arbor, MI 48109 USA (e-mail: Jingsun@umich.edu).

This is the author manuscript accepted for publication and has undergone full peer review but has not been through the copyediting, typesetting, pagination and proofreading process, which may lead to differences between this version and the Version of Record. Please cite this article as doi:

10.1002/rnc.3549 
optimization problem subject to nonlinear equality constraints [17]. In [18], we made use of control Lyapunov functions and the dual-mode approach to design a Lyapunov-based EMPC with a slight computational demand.

Motivated by the existing work in e.g. $[15,11,5]$, here we present a new stabilizing EMPC scheme with a contractive constraint for constrained nonlinear systems. Like other Lyapunov-based approaches, this scheme exploits the traditional terminal region and penalty function [3] to construct a contractive constraint. Sufficient conditions for guaranteed recursive feasibility and asymptotic stability are established. Moreover, the closed-loop transient performance evaluated over some finite-time intervals is analyzed under the same conditions. The proposed scheme has several special features.

(1) It asymptotically stabilizes the optimal steady state without additional strong duality or dissipativity conditions w.r.t the economic criterion, the conditions that are satisfied for linear systems with convex constraints and strictly convex costs, however, might not for other cases [5]. As a result, the proposed scheme can be used to optimized-based control of plants in general cases.

(2) This scheme makes use of a terminal inequality constraint and the original economic criterion. The notion of terminal inequality constraints is widely used in traditional MPC [3] and was first extended to EMPC [6] because it can increase the size of the feasible set of initial conditions and decrease the differences between predicted and closed-loop trajectories. However, in these (economic) MPC problems, cost functions are often modified by adding penalty functions to establish stability of the optimal steady state. Note that the optimal trajectories minimizing the original cost function are generally different from those minimizing a modified function. Hence, MPC closed-loop trajectories corresponding to the original and modified cost functions are not expected to be the same.

(3) The controller is computed by solving a single-layer optimization problem with a contractive constraint. Compared to the double-layer contractive EMPC [15], this scheme does not need the optimality of the contractive constraint and its stability is established only using contraction of the constraint.

(4) Recursive feasibility of this scheme always holds in the context of contractive constraints. It should be noted that to the best of our knowledge, the idea of imposing contractive constraints to MPC has been proposed for the first in [19-20] for traditional MPC of continuous-time systems, and in [15] for economic MPC of discrete-time systems, but with different assumptions, Lyapunov functions and optimization problem frameworks.

These features of the proposed EMPC provide an alternative to stabilizing EMPC design, and will be illustrated by a comparison study of an example for a continuous stirred tank reactor (CSTR). The rest of this paper is as follows. In Section 2 we describe the problem to be addressed. In Section 3 we propose the new EMPC scheme and establish some conditions under which recursive feasibility and asymptotic stability are guaranteed. Furthermore, we analyze transient economic performance of the proposed EMPC scheme in the same section. In Section 4 the proposed EMPC scheme is applied to a chemical reaction process. We conclude the paper in Section 5.

\section{PROBlem SetuP}

Notation. Let $R_{\geq 0}$ and $\mathbf{I}_{\geq 0}$ denote the sets of non-negative real and integer numbers, respectively. $\mathbf{I}_{a: b}$ is the set $\left\{i \in \mathbf{I}_{\geq 0}: a \leq i \leq b\right\}$ for some $a \in \mathbf{I}_{\geq 0}$ and $b \in \mathbf{I}_{\geq 0}$. Given two sets $S_{1} \subseteq R^{n}$ and $S_{2} \subseteq R^{n}$, define $S_{1} \backslash S_{2}=\left\{x \in R^{n} \mid x \in S_{1} \& x \notin S_{2}\right\}$. A function $\alpha: R^{n} \rightarrow R_{\geq 0}$ is positive definite w.r.t $x=s$ if it is continuous, $\alpha(s)=0$, and $\alpha(x)>0$ for all $x \neq s$. A continuous function $\alpha: R_{\geq 0} \rightarrow R_{\geq 0}$ is a class- $K$ function if it is strictly increasing and $\alpha(0)=0, \alpha(s)>0$ for all $s>0$; it is a class- $K_{\infty}$ function if it is a class- $K$ function and $\alpha(s) \rightarrow \infty$ as $s \rightarrow \infty$. A continuous function $\beta: R_{\geq 0} \times R_{\geq 0} \rightarrow R_{\geq 0}$ is a class- $K L$ function if $\beta(s, t)$ is a class- $K$ function in $s$ for each fixed $t \geq 0$; it is strictly decreasing in $t$ for every fixed $s>0$ and $\beta(s, t) \rightarrow 0$ as $t \rightarrow \infty$. For a given pair of functions $\alpha_{1}$ and $\alpha_{2}, \alpha_{1}{ }^{\circ} \alpha_{2}(s)$ denotes the function $\alpha_{1}\left(\alpha_{2}(s)\right)$ and $\alpha_{1}{ }^{k}(s)=$ $\alpha_{1}{ }^{\circ} \alpha_{1}{ }^{k-1}(s)$ for $k \in \mathbf{I}_{\geq 0}$, with $\alpha_{1}^{0}(s)=s$. The symbol ":=" denotes that the left-hand side of an equation is defined as the right-hand side. The converse applies to "=:".

Consider the discrete-time nonlinear systems of the form

$$
x_{k+1}=f\left(x_{k}, u_{k}\right), \quad \forall k \in \mathbf{I}_{\geq 0}
$$

where system state $x_{k} \in R^{n}$ and control input $u_{k} \in R^{m}$ at time $k$, and map $f: R^{n} \times R^{m} \rightarrow R^{n}$. It is assumed that the system has an equilibrium point $\left(x_{s}, u_{s}\right)$ such that $x_{s}=f\left(x_{s}, u_{s}\right)$. The solution of the system for a given sequence of control inputs $\mathbf{u}$ and initial state $x_{0}$ is denoted as $x_{k}=\varphi\left(k ; x_{0}, \mathbf{u}\right)$ for $k \in \mathbf{I}_{\geq 0}$, where $x_{0}=\varphi\left(0 ; x_{0}, \mathbf{u}\right)$. The system is subject to the state and control constraints

$$
x_{k} \in X, \quad u_{k} \in U, \quad \forall k \in \mathbf{I}_{\geq 0}
$$

where $X \subset R^{n}$ and $U \subset R^{m}$ are compact sets, containing the equilibrium point in their interior. We assume that the states can be measured at each time $k \in \mathbf{I}_{\geq 0}$. 
The economic criterion to be minimized is represented by the stage cost function $L_{e}: X \times U \rightarrow R$. The optimal operating point that stabilizes the system (1) is one of the steady-state points of (1), which is computed by

$$
\left(x_{s}, u_{s}\right)=\arg \min _{(x, u)}\left\{L_{e}(x, u) \mid x=f(x, u), x \in X, u \in U\right\} .
$$

For simplicity, we assume that $\left(x_{s}, u_{s}\right)$ is unique hereafter; otherwise, let $\left(x_{s}, u_{s}\right)$ denote any of the steady-state points satisfying (3).

In target-tracking MPC, $L_{e}(x, u)$ is often chosen as a positive definite function w.r.t $\left(x_{s}, u_{s}\right)$, i.e., $L_{e}(x, u) \geq 0$ for all $(x, u) \in X \times U$ and $L_{e}(x, u)=0$ if and only if $(x, u)=\left(x_{s}, u_{s}\right)$. In this case, the optimal operation often leads to closed-loop stability of $x_{s}$ using the standard MPC stability designs [3]. In EMPC, however, $L_{e}(x, u)$ is chosen according to some economic criteria such as energy saving, production cost and yield, etc. These economic criteria have to be minimized or maximized in terms of profits and environmental concerns for plants. Hence, in EMPC $L_{e}(x, u)$ is not necessarily positive definite w.r.t $\left(x_{s}, u_{s}\right)$. Consequently, convergence and stability properties in the optimal economic operation are in general not guaranteed using the standard MPC stability designs since these designs depend on the positive definiteness of $L_{e}(x, u)$.

The control problem of this paper consists in computing an MPC law by minimizing the economic objective function evaluated over a prediction horizon $0<N<\infty$,

$$
\sum_{i=0}^{N-1} L_{e}\left(x_{i}, u_{i}\right)
$$

subject to the constraints (1) and (2), which guarantees closed-loop asymptotic stability of the optimal operating point $\left(x_{s}, u_{s}\right)$. Moreover, we will investigate the transient performance of the closed-loop system

$$
J_{T}^{c l}(x)=\sum_{k=0}^{T} L_{e}\left(x_{k}, \mu_{N}\left(x_{k}\right)\right)
$$

for some finite-time interval $T \in \mathbf{I}_{\geq 1}$, where $\mu_{N}(x)$ is the first element of the control sequence minimizing (4) for $x_{0}=x$.

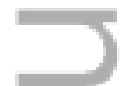

\section{CONTRACTIVE EMPC SCHEME}

Consider the system (1) and denote by $\left(x_{i \mid k}, u_{i \mid k}\right)$ the state and control input at time $k+i$, predicted at time $k$ for $i \in \mathbf{I}_{\geq 0}$. Let $\mathbf{u}=\left\{u_{0 \mid k}, u_{1 \mid k}, \ldots, u_{N-1 \mid k}\right\}$ be a sequence of $N$ predicted control inputs and $\mathbf{x}=\left\{x_{0 \mid k}, x_{1 \mid k}, \ldots, x_{N \mid k}\right\}$ be its corresponding predicted state trajectory according to the model (1). According to the economic criterion $L_{e}(x, u)$, we define the cost function to be minimized, $J_{N}$, as

$$
J_{N}\left(x_{k}, \mathbf{u}\right)=\sum_{i=0}^{N-1} L_{e}\left(x_{i \mid k}, u_{i \mid k}\right)
$$

where $x_{k}$ is the state at the current time $k$ and $x_{0 \mid k}=x_{k}$.

Let $J_{a}: X \times U^{N} \rightarrow R_{\geq 0}$ be an auxiliary cost function and $\eta \in R_{\geq 0}$ be a scalar, which will be specified later on. We propose a new contractive EMPC formulation in which the following finite horizon optimal control problem is solved at each time $k \in \mathbf{I}_{\geq 0}$ :

$$
\begin{aligned}
& J_{N}^{*}\left(x_{k}\right)=\min _{\mathbf{u}} J_{N}\left(x_{k}, \mathbf{u}\right) \\
\text { s.t. } & x_{i+1 \mid k}=f\left(x_{i \mid k}, u_{i \mid k}\right), \quad \forall i \in \mathbf{I}_{0: N-1} \\
& x_{i+1 \mid k} \in X, u_{i \mid k} \in U, \quad \forall i \in \mathbf{I}_{0: N-1} \\
& x_{0 \mid k}=x_{k} \in X \\
& x_{N \mid k} \in X_{f} \\
& J_{a}\left(x_{k}, \mathbf{u}\right) \leq \eta_{k}
\end{aligned}
$$$$
\text { ( }
$$

where $J_{N}^{*}(x)$ is the optimal value function of (7) and terminal region $X_{f}$ is a compact subset of $X$. The constraint (7d) is called the initial condition and (7e) is the terminal constraint. Here the constraint (7f) will be designed as a contractive constraint used to establish closed-loop stability of $\left(x_{s}, u_{s}\right)$. We denote the (possible local) optimal solution to the optimization problem as $\mathbf{u}^{*}\left(x_{k}\right)=\left\{u_{0 \mid k}{ }^{*}\left(x_{k}\right), \ldots, u_{N-1 \mid k}{ }^{*}\left(x_{k}\right)\right\}$ and then $J_{N}{ }^{*}\left(x_{k}\right)=J_{N}\left(x_{k}, \mathbf{u}^{*}\left(x_{k}\right)\right)$.

Consider the system (1) with the constraint (2). We define the set of admissible $(x, \mathbf{u})$ pairs as

$$
Z_{N}=\left\{(x, \mathbf{u}) \mid \varphi(k ; x, \mathbf{u}) \in X, u_{k} \in U, \varphi(N ; x, \mathbf{u}) \in X_{f}, \forall k \in \mathbf{I}_{0: N-1}\right\}
$$

where $x$ is an initial state at initial time $k=0$ and $\mathbf{u}=\left\{u_{0}, \ldots, u_{N-1}\right\}$. Then the set of admissible initial states, $X_{N}$, is defined as the projection of $Z_{N}$ onto $X$, i.e.,

$$
X_{N}=\left\{x \in X \mid \exists \mathbf{u} \in R^{m N} \text { such that }(x, \mathbf{u}) \in Z_{N}\right\} .
$$

At time $k \in \mathbf{I}_{\geq 0}$, for the state $x_{k} \in X_{N}$ the set of admissible control sequences, $U_{N}$, is defined as 


$$
U_{N}\left(x_{k}, \eta_{k}\right)=\left\{\mathbf{u} \in R^{m N} \mid\left(x_{k}, \mathbf{u}\right) \in Z_{N},(7 \mathrm{f})\right\} .
$$

Clearly, the optimal solution to (7) satisfies $\mathbf{u}^{*}\left(x_{k}\right) \in U_{N}\left(x_{k}, \eta_{k}\right)$.

We now construct the function $J_{a}(x, \mathbf{u})$ and scalar $\eta$. To this end, we introduce two auxiliary functions $L_{a}$ : $X \times U \rightarrow R_{\geq 0}$ and $E_{a}: X \rightarrow R_{\geq 0}$, where $L_{a}$ is positive definite w.r.t $\left(x_{s}, u_{s}\right)$ and $E_{a}$ is positive definite w.r.t $x_{s}$. We define $J_{a}(x, \mathbf{u})$ as follows:

$$
J_{a}\left(x_{k}, \mathbf{u}\right)=E_{a}\left(x_{N \mid k}\right)+\sum_{i=0}^{N-1} L_{a}\left(x_{i \mid k}, u_{i \mid k}\right)
$$

where $x_{i \mid k}=\varphi\left(k+i ; x_{k}, \mathbf{u}\right)$ for $i \in \mathbf{I}_{1: N-1}$ with $x_{0 \mid k}=x_{k}$ and $\mathbf{u} \in U_{N}\left(x_{k}, \eta_{k}\right)$ corresponding to the state $x_{k}$ at time $k \in \mathbf{I}_{\geq 0}$. Since $L_{a}(x, u)$ and $E_{a}(x)$ are positive definite w.r.t $\left(x_{s}, u_{s}\right)$ and $x_{s}$, we have $J_{a}\left(x_{k}, \mathbf{u}\right) \geq L_{a}\left(x_{0 \mid k}, u_{0 \mid k}\right) \geq 0$ with $\mathbf{u} \in U_{N}\left(x_{k}, \eta_{k}\right)$ for any $x_{k} \in X_{N}$. Substituting $\mathbf{u}^{*}\left(x_{k}\right)$ into (11), we have a value function of $J_{a}\left(x_{k}, \mathbf{u}\right)$ as follows:

$$
V\left(x_{k}\right)=E_{a}\left(x_{N \mid k}^{*}\right)+\sum_{i=0}^{N-1} L_{a}\left(x_{i \mid k}^{*}, u_{i \mid k}^{*}\left(x_{k}\right)\right)
$$

where $x_{i k}{ }^{*}=\varphi\left(k+i ; x_{k}, \mathbf{u}^{*}\left(x_{k}\right)\right)$ for $i \in \mathbf{I}_{1: N-1}$. Note that $\mathbf{u}^{*}\left(x_{k}\right)$ is the optimal control for (7) and not for (11). Clearly, we have $V\left(x_{k}\right) \geq L_{a}\left(x_{k}, u_{0 \mid k}{ }^{*}\left(x_{k}\right)\right) \geq 0$ for all $x_{k} \in X_{N}$ due to the positive definiteness of $L_{a}(x, u)$ and $E_{a}(x)$. To obtain $\eta$, we need an assumption on the terminal region $X_{f}$ and the functions $L_{a}$ and $E_{a}$.

Assumption 1: There exist a compact terminal region $X_{f} \subset X$, containing $x_{s}$ in its interior, and a continuous control law $\mu_{f}: X_{f} \rightarrow U$, with $\mu_{f}\left(x_{s}\right)=u_{s}$, such that

$$
E_{a}\left(f\left(x, \mu_{f}(x)\right)\right)-E_{a}(x) \leq-L_{a}\left(x, \mu_{f}(x)\right), \forall x \in X_{f} .
$$

Assumption 1 implies that the region $X_{f}$ is an invariant set of the system (1) in closed-loop with $\mu_{f}(x)$ provided that $X_{f}$ is a sublevel set of $E_{a}(x)$. In the literature, many approaches have been proposed to design $\mu_{f}(x)$ as well as $X_{f}$ and $E_{a}(x)$ satisfying this assumption; see, e.g., [21-24].

Let $\mathbf{u}^{*}\left(x_{k-1}\right)$ be the optimal solution to (7) at time $k-1$ and construct a sequence at time $k$ as

Then we define $\eta_{k}$ as

$$
\hat{\mathbf{u}}_{k}=\left\{u_{1 \mid k-1}^{*}\left(x_{k-1}\right), \cdots, u_{N-1 \mid k-1}^{*}\left(x_{k-1}\right), \mu_{f}\left(x_{N \mid k-1}^{*}\right)\right\} \text {. }
$$

$$
\eta_{k}=V\left(x_{k-1}\right)+\beta\left[J_{a}\left(x_{k}, \hat{\mathbf{u}}_{k}\right)-V\left(x_{k-1}\right)\right]
$$

with some $\beta \leq 1$. Since $V\left(x_{k}\right) \geq 0$ and $J_{a}\left(x_{k}, \mathbf{u}\right) \geq 0$ for any $x_{k} \in X_{N}$ and $\mathbf{u} \in U_{N}\left(x_{k}, \eta_{k}\right)$, it is straightforward to obtain that $\eta_{k} \geq 0$ for all $k \in \mathbf{I}_{>0}$. Note that although $\eta_{k}$ depends on $x_{k-1}, V\left(x_{k}\right)$ is the function on $x_{k}$ due to the receding horizon control principle.

In MPC, the control input applied to the plant is the first action of $\mathbf{u}^{*}\left(x_{k}\right)$, which yields an implicit state feedback EMPC law $\mu_{N}(x)$ defined as

$$
\mu_{N}\left(x_{k}\right)=u_{0 \mid k}^{*}\left(x_{k}\right), \quad \forall k \in \mathbf{I}_{\geq 0} .
$$

This controller gives rise to the closed-loop system

$$
x_{k+1}=f\left(x_{k}, \mu_{N}\left(x_{k}\right)\right), \quad \forall k \in \mathbf{I}_{\geq 0} .
$$

The procedure for implementing the EMPC controller (16) is summarized as Algorithm 1.

Algorithm 1: (Economic MPC with a contractive constraint)

1) (Initialization) Pick $N>1, \beta \leq 1$ and $L_{a}(x, u)$, and compute $\left(E_{a}, X_{f}, \mu_{f}\right)$ to satisfy (13); set $\eta_{0}:=+\infty$ for a given $x_{0} \in X_{N}$ and let $k=0$. Solve the optimization problem (7) and obtain its optimal solution $\mathbf{u}^{*}\left(x_{0}\right)$. Implement the control action $u_{0}=\mu_{N}\left(x_{0}\right)$ to the system (1) and let $k=1$.

2) Compute $V\left(x_{k-1}\right)$ and evaluate $\eta_{k}$ by (14)-(15) with the state $x_{k}$ at time $k$.

3) Solve the optimization problem (7) and obtain its optimal solution $\mathbf{u}^{*}\left(x_{k}\right)$.

4) Implement the control action $u_{k}=\mu_{N}\left(x_{k}\right)$ to the system (1).

5) Let $k=k+1$ and go back to Step 2 .

Note that in Algorithm 1 the contractive constraint (7f) is inactive at initial time $k=0$ since $\eta_{0}:=+\infty$. Hence, the set $X_{N}$ defined as (9) is identified as the set of admissible initial states of the optimization problem (7).

Lemma 1: Consider Assumption 1 and the parameter $\beta \leq 1$. The optimization problem (7) is recursively feasible within the invariant set $X_{N}$.

Proof: Let $\mathbf{u}^{*}\left(x_{k}\right)$ be the optimal solution to (7) at time $k$, with $x_{0 \mid k}=x_{k} \in X_{N}$. At the next time $k+1$, we use (14) to choose a candidate sequence and a corresponding state sequence as follows:

$$
\begin{gathered}
\hat{\mathbf{u}}_{k+1}=\left\{u_{1 \mid k}^{*}\left(x_{k}\right), \cdots, u_{N-1 \mid k}^{*}\left(x_{k}\right), \mu_{f}\left(x_{N \mid k}^{*}\right)\right\} \\
\hat{\mathbf{x}}_{k+1}=\left\{x_{|| k}^{*}, \cdots, x_{N \mid k}^{*}, x_{N+1 \mid k}\right\}
\end{gathered}
$$


where $x_{i \mid k}{ }^{*}=\varphi\left(k+i ; x_{k}, \mathbf{u}^{*}\left(x_{k}\right)\right) \in X$ for $i \in \mathbf{I}_{1: N}$ and $x_{N+1 \mid k}=f\left(x_{N \mid k}{ }^{*}, \mu_{f}\left(x_{N \mid k}{ }^{*}\right)\right)$. Due to the terminal constraint (7e) and the invariance property of $X_{f}$, we have $x_{N+1 \mid k} \in X_{f}$. Thus, the constraints in (7b)-(7e) are fulfilled.

Now we consider the contractive constraint (7f). Since this constraint is inactive at $k=0$, we test it for $k \in \mathbf{I}_{\geq 1}$. In order to satisfy (7f), we substitute (18)-(19) to (11) and consider the following inequality:

$$
J_{a}\left(x_{k+1}, \hat{\mathbf{u}}_{k+1}\right) \leq \eta_{k+1}=V\left(x_{k}\right)+\beta\left[J_{a}\left(x_{k+1}, \hat{\mathbf{u}}_{k+1}\right)-V\left(x_{k}\right)\right] .
$$

Let $\alpha=1-\beta \geq 0$. Then (20) can be rewritten as

$$
J_{a}\left(x_{k+1}, \hat{\mathbf{u}}_{k+1}\right) \leq J_{a}\left(x_{k+1}, \hat{\mathbf{u}}_{k+1}\right)+\alpha\left[V\left(x_{k}\right)-J_{a}\left(x_{k+1}, \hat{\mathbf{u}}_{k+1}\right)\right] .
$$

From Assumption 1, it is known that

$$
V\left(x_{k}\right)-J_{a}\left(x_{k+1}, \hat{\mathbf{u}}_{k+1}\right) \geq L_{a}\left(x_{k}, \mu_{N}\left(x_{k}\right)\right) \geq 0
$$

Hence, the inequality (21) holds if $\alpha \geq 0$ and the equality is always true if $\alpha=0$. Therefore, the inequality (20) holds for any $\beta \leq 1$. This implies that (18)-(19) satisfy (7f) and the candidate (18) satisfies the overall constraints in (7), which implies that this candidate is a feasible solution to (7) at time $k+1$. Hence, $U_{N}\left(x_{k+1}, \eta_{k+1}\right)$ is not empty and $x_{k+1}=f\left(x_{k}, \mu_{N}\left(x_{k}\right)\right) \in X_{N}$ for $\forall x_{k} \in X_{N}$. This establishes recursive feasibility of (7) with the invariant set $X_{N}$.

Note that similar to the standard MPC ${ }^{[3,21]}$, a larger size of $X_{f}$ generally leads to a larger size of $X_{N}$ due to the property of the terminal region constraint.

Remark 1: It is possible that $V(x)>J_{a}(x, \hat{\mathbf{u}})$ since $\mathbf{u}^{*}(x)$ is not optimal for $J_{a}(x, \mathbf{u})$ but for $J_{N}(x, \mathbf{u})$. Moreover, the optimal path from $x_{0}=x_{s}$ to $x_{N}=x_{s}$ is often different from $x_{k} \equiv x_{s}$ for all $k \in \mathbf{I}_{0: N}$ in the context of EMPC [4]. Consequently, the function $V(x)$ is not necessarily positive definite w.r.t $x_{s}$ as it is in target-tracking MPC. In other words, it is possible that $V\left(x_{s}\right) \neq 0$ unless some additional conditions are imposed. Note that $(x, u)=\left(x_{s}, u_{s}\right)$ if $V(x)=0$ due to the positive definiteness of $L_{a}$ and $E_{a}$ w.r.t $\left(x_{s}, u_{s}\right)$ and $x_{s}$, respectively.

Assumption 2: The functions $f$ and $L_{e}$ are continuous on the compact sets $X \times U$, respectively, and there exist some class- $K_{\infty}$ functions $\alpha_{f}, \alpha_{l}$, and $\gamma_{i}, i=1, \ldots, 4$ such that $\|f(x, u)-f(z, v)\| \leq \alpha_{f}(\|(x, u)-(z, v)\|),\left\|L_{e}(x, u)-L_{e}(z, v)\right\| \leq \alpha_{l}(\|(x, u)-$ $(z, v) \|), L_{a}(x, u) \leq \gamma_{1}\left(\left\|x-x_{s}\right\|\right)+\gamma_{2}\left(\left\|u-u_{s}\right\|\right)$, and $\gamma_{3}\left(\left\|x-x_{s}\right\|\right) \leq E_{a}(x) \leq \gamma_{4}\left(\left\|x-x_{s}\right\|\right), \forall(x, u),(z, v) \in X \times U$ for some vector norm $\|\cdot\|$.

Since $L_{a}$ and $E_{a}$ are positive definite w.r.t. $\left(x_{s}, u_{s}\right)$ and $x_{s}$, respectively, the class- $K_{\infty}$ functions $\gamma_{i}, i=1, \ldots, 4$ exist in the finite dimensional case with $X$ and $U$, and with continuity of $L_{a}$ and $E_{a}$.

Assumption 3: The optimal solution to (7), $\mathbf{u}^{*}(x)$, satisfies that $u_{i \mid k}{ }^{*}\left(x_{s}\right)=u_{s}$ for $i \in \mathbf{I}_{0: N-1}$. Moreover, there exists a class- $K_{\infty}$ function $\alpha_{u}$ such that $\left\|u_{i \mid k}{ }^{*}(x)-u_{i \mid k}{ }^{*}\left(x_{s}\right)\right\| \leq \alpha_{u}\left(\left\|x-x_{s}\right\|\right)$ for any $x \in X_{f}$ and $i \in \mathbf{I}_{0: N-1}$.

Assumption 3 holds if $\mathbf{u}^{*}(x)$ is continuous on $X_{f}$. As a candidate solution to (7), the sequence $\mathbf{u}(x)$ with $u_{i \mid k}(x)=u_{f}(x)$ for $i \in \mathbf{I}_{0: N-1}$ satisfies Assumption 3. Combining Assumptions 1 3, we have the following stability result.

Theorem 1: Suppose that Assumptions 1-3 hold and the parameter $0<\beta \leq 1$. If (7) is initially feasible, then $x_{s}$ is an asymptotically stable equilibrium point of the closed-loop system (17) with the region of attraction $X_{N}$.

Proof: By applying Lemma 1 and the assumptions recursively, it is obtained that (7) is feasible for $x_{k} \in X_{N}$ at time $k \in \mathbf{I}_{\geq 0}$. Thus, $X_{N}$ is an invariant region of the closed-loop system.

Let $\mathbf{u}^{*}\left(x_{k}\right)$ and $\mathbf{u}^{*}\left(x_{k+1}\right)$ be the optimal solutions to (7) at time $k$ and $k+1$, respectively. Consider a candidate Lyapunov function $V(x)$ given as (12). For all $x_{k} \in X_{N}$, the constraint (7f) and the definition of $V(x)$ lead to

$$
V\left(x_{k+1}\right)-V\left(x_{k}\right) \leq \beta\left[J_{a}\left(x_{k+1}, \hat{\mathbf{u}}_{k+1}\right)-V\left(x_{k}\right)\right] \leq-\beta L_{a}\left(x_{k}, \mu_{N}\left(x_{k}\right)\right)
$$

where $\hat{\mathbf{u}}_{k+1}$ is given by (18). Due to the positive definiteness of $L_{a}(x, u)$ w.r.t $\left(x_{s}, u_{s}\right), \beta L_{a}(x, u) \geq \rho_{1}\left(\left\|x-x_{s}\right\|\right)$ for all $(x, u) \in$ $X \times U$, where $\rho_{1}$ is a class $-K_{\infty}$ function. Hence, $V(x)$ is a strictly monotone decreasing function along the trajectories of (17) and $V\left(x_{k}\right) \leq V\left(x_{0}\right)$ for all $k \in \mathbf{I}_{\geq 0}$. Moreover, by (23) we have $V(x) \geq \rho_{1}\left(\left\|x-x_{s}\right\|\right)$ since $V(x) \geq 0$ for all $x \in X_{N}$.

In order to obtain the upper bound of $V(x)$, we separately consider the cases of $x \in X \backslash X_{f}$ and $x \in X_{f}$. Let $L_{\max }=\max$ $\{L(x, u) \mid(x, u) \in X \times U\}$ and $E_{\max }=\max \{E(x) \mid x \in X\}$. Then for the state $x \in X \backslash X_{f}$, it holds that $V(x) \leq E_{\max }+N L_{\max }=: V_{\max }$.

Considering any state $x \in X_{f}$ at time $k$, i.e., $x_{k}=x$, it is obtained from Assumptions 2 and 3 that

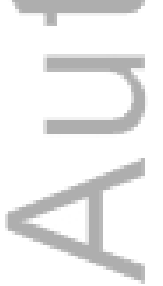

$$
\begin{aligned}
V(x) \leq & \gamma_{4}\left(\left\|x_{N \mid k}^{*}-x_{s}\right\|\right)+\sum_{i=0}^{N-1}\left[\gamma_{1}\left(\left\|x_{i \mid k}^{*}-x_{s}\right\|\right)+\gamma_{2}\left(\left\|u_{i \mid k}^{*}(x)-u_{s}\right\|\right)\right] \\
= & \gamma_{4}\left(\left\|f\left(x_{N-1 \mid k}^{*}, u_{N-1 \mid k}^{*}\right)-f\left(x_{s}, u_{s}\right)\right\|\right)+\gamma_{1}\left(\left\|x_{0 \mid k}-x_{s}\right\|\right)+ \\
& \sum_{i=1}^{N-1} \gamma_{1}\left(\left\|f\left(x_{i \mid k}^{*}, u_{i \mid k}^{*}\right)-f\left(x_{s}, u_{s}\right)\right\|\right)+\sum_{i=0}^{N-1} \gamma_{2}\left(\left\|u_{i \mid k}^{*}(x)-u_{i \mid k}^{*}\left(x_{s}\right)\right\|\right) \\
\leq & \gamma_{4} \circ \alpha_{N}\left(\left\|x-x_{s}\right\|\right)+N \gamma_{2} \circ \alpha_{u}\left(\left\|x-x_{s}\right\|\right)+\sum_{i=0}^{N-1} \gamma_{1} \circ \alpha_{i}\left(\left\|x-x_{s}\right\|\right) \\
= & : \rho\left(\left\|x-x_{s}\right\|\right) .
\end{aligned}
$$


where $\alpha_{0}(r)=r$ and $\alpha_{i}(r)=\alpha_{f}^{\circ} \alpha_{i-1}(r)+\alpha_{f} \alpha_{u}(r) \in K_{\infty}$ for $i \in \mathbf{I}_{1: N}$. Clearly, $\rho$ is a class- $K_{\infty}$ function. If $\rho\left(\left\|x-x_{s}\right\|\right) \geq V_{\max }$ for $x \in X \backslash X_{f}$, we get $V(x) \leq \rho\left(\left\|x-x_{s}\right\|\right)$ for all $x \in X$. Otherwise, we multiply $\rho\left(\left\|x-x_{s}\right\|\right)$ by a constant $C$ such that $C \rho\left(\left\|x-x_{s}\right\|\right) \geq$ $V_{\max }$ for $x \in X \backslash X_{f}$. This operation yields $V(x) \leq \rho_{2}\left(\left\|x-x_{s}\right\|\right)$ for all $x \in X$, where $\rho_{2}(r):=\max \{1, C\} \rho(r)$. Hence, we have $\rho_{1}\left(\left\|x-x_{s}\right\|\right) \leq V(x) \leq \rho_{2}\left(\left\|x-x_{s}\right\|\right)$ for all $x \in X_{N}$, with class- $K_{\infty}$ functions $\rho_{1}$ and $\rho_{2}$. Combining the inequality $(23), V(x)$ is a Lyapunov function of the closed-loop system (17) and $x_{s}$ is an asymptotically stable equilibrium point of (17) within $X_{N}$. Since $X_{N}$ is invariant for (17), it is a region of attraction of the closed-loop system.

Remark 2: From the proof of Lemma 1 and Theorem 1, one can see that the contractive property of (7f) is crucial in guaranteeing stability of the optimal steady state of the closed-loop system. Hence, the resulting EMPC (here referred to as Contractive EMPC) scheme can be viewed as a variant of contractive MPC [19,20,25], but with several different features. In traditional contractive MPC [3], a Lyapunov function is chosen a priori as a positive definite function $M(x)$, e.g., $M(x)=x^{\mathrm{T}} P x$ with $P>0$, to ensure that $M(x(k ; x, \mathbf{u})) \leq M(x)$ with $k=1,2, \ldots, N$. Then a variable horizon $\left(N^{*}\right)$ optimal control problem is solved online, where $X_{f}$ depends on the current state $x$ and $M(x)$. In the original version of contractive MPC, the whole control sequence $\mathbf{u}^{*}$ is applied to plants in an open-loop fashion and the procedure is repeated at every time interval $N^{*}$. Under assumptions of feasibility, exponential stability of the origin is ensured by the contractive constraint. Unlike the traditional contractive MPC, here the Lyapunov function is defined as $V(x)=J_{a}\left(x, \mathbf{u}^{*}(x)\right)$ and it is not necessarily positive definite w.r.t $x_{s}$ since $\mathbf{u}^{*}(x)$ is not optimal for $J_{a}(x, \mathbf{u})$ but for $J_{N}(x, \mathbf{u})$. Moreover, the optimization (7) is a fixed horizon problem where $X_{f}$ is chosen to satisfy Assumption 1, i.e., $X_{f}$ is not related to the current state $x$ and $V(x)$, and recursive feasibility is always guaranteed. The controller obtained here follows the standard receding horizon principle, in the sense that only the first control element of the solution to (7) is applied to (1) at each time. In addition, here the contractive constraint (7f) does not lead to exponential stability of the closed-loop system (17).

Remark 3: In the context of EMPC, the idea of imposing a contractive property on the closed-loop behavior is also presented in [15]. In that work, the author regarded stability and economics as two conflicting objectives and proposed a double-layer lexicographic optimization formulation, i.e.

$\begin{aligned} 1^{s t} \text { layer: }: & \left\{\begin{array}{l}\mathbf{u}_{1}^{*}\left(x_{k}\right)=\arg \min _{\mathbf{u}}\left\{J_{t r}\left(x_{k}, \mathbf{u}\right)=\sum_{i=0}^{N-1} L_{a}\left(x_{i \mid k}, u_{i \mid k}\right)\right\} \\ \text { s.t. } \quad(7 b)-(7 d), x_{N \mid k}=x_{s}\end{array}\right. \\ 2^{\text {nd }} \text { layer: } & \left\{\begin{array}{l}\mathbf{u}_{2}^{*}\left(x_{k}\right)=\arg \min _{\mathbf{u}} J_{N}\left(x_{k}, \mathbf{u}\right) \\ \text { s.t. } \quad(7 b)-(7 d), x_{N \mid k}=x_{s} \\ J_{t r}\left(x_{k}, \mathbf{u}\right) \leq \xi_{k}:=J_{t r}^{*}\left(x_{k}\right)+a\left[V_{t r}\left(x_{k-1}\right)-J_{t r}^{*}\left(x_{k}\right)\right]\end{array}\right.\end{aligned}$

where $J_{t r}(x, \mathbf{u})$ is the target-tracking function, $J_{N}(x, \mathbf{u})$ is the economic function given as $(6), J_{t r}{ }^{*}(x)=J_{t r}\left(x, \mathbf{u}_{1}{ }^{*}(x)\right)$, $V_{t r}(x)=J_{t r}\left(x, \mathbf{u}_{2}{ }^{*}(x)\right)$, and some $0 \leq a<1$. The last constraint in (25), i.e., $J_{t r}(x, \mathbf{u}) \leq \xi$, is a contractive constraint, which, together with inherent robustness of target-tracking MPC, ensures that $V_{t r}(x)$ is a Lyapunov function of the system (1) in closed-loop with this EMPC law specified as the first element of $\mathbf{u}_{2}{ }^{*}(x)$. Due to the global optimality of $\mathbf{u}_{1}{ }^{*}(x)$ and the positive definiteness of $J_{t r}{ }^{*}(x)$ w.r.t $x_{s}, V_{t r}(x)$ is positive definite w.r.t $x_{s}$. Note that this formulation needs to online solve two non-convex, nonlinear optimization problems, which significantly increases the computational load of implementing MPC [14]. In particular, non-convexity may lead to non-global solution in both layers and therefore, no guarantee of feasibility for (25) due to discontinuous Pareto fronts of non-convex multiobjective optimization [15]. Finally, the terminal equality will reduce the size of feasible set of initial conditions [6].

Note that the contractive constraint (7f) can be replaced with some conditions, e.g., [26]

$$
J_{a}\left(x_{k}, \mathbf{u}\right)-V\left(x_{k-1}\right) \leq-\sigma L_{a}\left(x_{k-1}, \mu_{N}\left(x_{k-1}\right)\right)
$$

for some $\sigma \in R_{\geq 0}$. In this case, recursive feasibility and stability also hold for $\sigma \in(0,1]$ from the proof of Lemma 1 and Theorem 1. Additionally, since recursive feasibility of (7), which is essential in establishing stability, is ensured for $\beta \in(0,1]$ but not for $\beta>1$, stability of (17) cannot be established for the general case of $\beta>1$. But on a case by case it is possible to find conditions for $\beta>1$ to establish stability of (17).

Let the balls $B_{r}$ be defined as $B_{r}=\left\{x \in X:\left\|x-x_{s}\right\| \leq r\right\}$ for some norm $\|\cdot\|$ and $r>0$, and the level sets of $V(x)$ as $S_{c}=$ $\{x \in X: V(x) \leq c\}$ for some $c>0$. Since the system (17) asymptotically converges to $\left(x_{s}, u_{s}\right)$ while remaining bounded for all $k \in \mathbf{I}_{\geq 0}$, for a small $c>0$ there exists a finite time interval $T_{c} \in \mathbf{I}_{\geq 1}$ such that for all $k \geq T_{c}, \varphi\left(k ; x_{0}, \mu_{N}\right) \in S_{c}$ with $x_{0} \in X_{N}$. Then from Theorem 1 we have the following corollary on $V(x)$ and the terminal predicted state $x_{N \mid k}$.

Corollary 1: Consider a small number $c>0$ such that $S_{c} \subset X_{f}$. Under the assumptions in Theorem 1, the closed-loop states of (17) enter $S_{c}$ in such way that $V(x)$ decreases exponentially. Moreover, if the state $x_{k} \in X_{N} \mid S_{c}$, then its optimal 
predicted state $x_{N \mid k}{ }^{*}$ satisfies that

$$
\left\|x_{N \mid k}^{*}-x_{s}\right\| \leq \theta_{1}\left(\left\|x_{0}-x_{s}\right\|, k\right)
$$

where $\theta_{1}$ is a class- $K L$ function.

Proof: Let $\hat{S}$ be the closure of set $X_{N} S_{c}$. We compute the minimal value of $L_{a}(x, u) / J_{a}(x, \mathbf{u})$ for $\forall(x, \mathbf{u}) \in\left(\hat{S}, U^{N}\right)$ and denote it as $\bar{a}$, where $u$ is the first element of sequence $\mathbf{u}$. Note that $\bar{a}$ exists due to Assumptions 1-3 and the positive definiteness of $L_{a}(x, u)$ and $E_{a}(x, u)$. Clearly, we have $0<\bar{a}<1$. By the inequality (23), we have $V\left(x_{k+1}\right)-V\left(x_{k}\right) \leq-\bar{a} \beta V\left(x_{k}\right)$. This implies that $V\left(x_{k}\right) \leq(1-\bar{a} \beta)^{k} V\left(x_{0}\right)$. Since $e^{(s-1)}-s \geq 0 \Leftrightarrow s^{k} \leq e^{(s-1) k}$ for $\forall s \in[0,1)$ and $k \in \mathbf{I}_{\geq 0}$, we have

$$
V\left(x_{k}\right) \leq V\left(x_{0}\right) e^{-(\bar{a} \beta) k}, \quad \forall x_{k} \in X_{N} \backslash S_{c} .
$$

Since $S_{c}$ is the sublevel set of $V(x)$ and the system (17) is asymptotically stable, $V(x)$ decreases exponentially until $V(x) \leq c$, i.e., $x \in S_{c}$.

By Assumption 2 and the definition of $V(x)$, it is derived that $\gamma_{3}\left(\left\|x_{N \mid k}{ }^{*}-x_{s}\right\|\right) \leq E_{a}\left(x_{N \mid k}{ }^{*}\right) \leq V\left(x_{k}\right)$ for $\forall x_{k} \in X_{N}$. Consider any state $x_{k} \in X_{N} \backslash S_{c \text { s }}$ Then combining (28), we have

$$
=\left\|x_{N \mid k}^{*}-x_{s}\right\| \leq \gamma_{3}^{-1}\left(\rho_{2}\left(\left\|x_{0}-x_{s}\right\|\right) e^{-(\bar{a} \beta) k}\right)=: \theta_{1}\left(\left\|x_{0}-x_{s}\right\|, k\right)
$$

for $\forall x_{k} \in X_{N} S_{c}$ of (17). Since the functions $\gamma_{3}, \rho_{2} \in K_{\infty}, \theta_{1}$ is a class- $K L$ function and thus (27) holds.

Remark 4: It is observed from (28) that the larger the value of $\beta$, the faster the decaying of $V(x)$. This implies that a lager value of $\beta$ speeds up the closed-loop system (17) to approach to the optimal steady-state point. In this sense, $\beta$ is named as the contractive factor reflecting the contractive property of the closed-loop behavior.

Now we analyze economic performance of the proposed EMPC scheme. Without loss of generality, here we assume that $L_{e}(x, u)>0$ for all $x \in X$ and $u \in U$. Given an initial state $x_{0} \in X_{N} S_{c}$, from Corollary 1 we can find a finite time interval $T>0$ such that $\varphi\left(k ; x_{0}, \mu_{N}(\cdot)\right) \in X_{N} S_{c}$ for $k \in \mathbf{I}_{1: T}$ and $\varphi\left(k ; x_{0}, \mu_{N}(\cdot)\right) \in S_{c}$ for $k \in \mathbf{I}_{\geq T+1}$. Moreover, we consider any admissible control sequence $\mathbf{u}$ and its associated trajectory $\varphi\left(k ; x_{0}, \mathbf{u}\right)$ which satisfies $\left\|\varphi\left(T ; x_{0}, \mathbf{u}\right)-x_{s}\right\| \leq$ $\left\|\varphi\left(T ; x_{0}, \mu_{N}(\cdot)\right)-x_{s}\right\| \leq r$ for some $r>0$. Then we have the following results on the transient performance (5) evaluated over the finite time interval $T$.

Proposition 1: Consider a small number $c>0$ such that $S_{c} \subset X_{f}$. Under the assumptions in Theorem 1, the transient performance (5) evaluated over the finite time interval $T$ satisfies that

$$
J_{T}^{c l}\left(x_{0}\right) \leq J_{T}\left(x_{0}, \mathbf{u}\right)+(N-T) \alpha_{L} \circ \rho_{2}^{-1}(c)+N L_{e}\left(x_{s}, u_{s}\right)+\sum_{k=0}^{T-1} \theta_{2}\left(\left\|x_{0}-x_{s}\right\|, k\right) .
$$

where $\alpha_{L}$ is a class- $K_{\infty}$ function and $\theta_{2}$ is a class- $K L$ function.

Proof: Let the sequences $\mathbf{u}^{*}\left(x_{k}\right)$ and $\mathbf{u}^{*}\left(x_{k+1}\right)$ be the optimal solutions to (7) at time $k$ and $k+1$, respectively, where $x_{k+1}=f\left(x_{k}, \mu_{N}\left(x_{k}\right)\right)$. Considering the sequence (18), we compute the optimal value functions of $J_{N}$ as follows:

$$
\begin{gathered}
J_{N}^{*}\left(x_{k}\right)=L_{e}\left(x_{k}, \mu_{N}\left(x_{k}\right)\right)+\sum_{i=1}^{N-1} L_{e}\left(x_{i \mid k}^{*}, u_{i \mid k}^{*}\right) \\
J_{N}^{*}\left(x_{k+1}\right) \leq J_{N}\left(x_{k+1}, \hat{\mathbf{u}}_{k+1}\right)=L_{e}\left(x_{N \mid k}^{*}, \mu_{f}\left(x_{N \mid k}^{*}\right)\right)+\sum_{i=0}^{N-2} L_{e}\left(x_{i+|| k}^{*}, u_{i+|| k}^{*}\right)
\end{gathered}
$$

which lead to

$$
J_{N}^{*}\left(x_{k+1}\right)-J_{N}^{*}\left(x_{k}\right) \leq L_{e}\left(x_{N \mid k}^{*}, \mu_{f}\left(x_{N \mid k}^{*}\right)\right)-L_{e}\left(x_{k}, \mu_{N}\left(x_{k}\right)\right) .
$$

Adding (31) from $k=0$ to $k=T-1$, it is obtained that

$$
J_{T}^{c l}\left(x_{0}\right) \leq J_{N}^{*}\left(x_{0}\right)-J_{N}^{*}\left(x_{T}\right)+\sum_{k=0}^{T-1} L_{e}\left(x_{N \mid k}^{*}, \mu_{f}\left(x_{N \mid k}^{*}\right)\right) \leq J_{N}^{*}\left(x_{0}\right)+\sum_{k=0}^{T-1} L_{e}\left(x_{N \mid k}^{*}, \mu_{f}\left(x_{N \mid k}^{*}\right)\right) .
$$

By Assumption 2, for $x \in X_{f}$ we have $L_{e}\left(x, \mu_{f}(x)\right)-L_{e}\left(x_{s}, u_{s}\right) \leq \alpha_{l}\left(\left\|x-x_{s}\right\|\right)+\alpha_{l}{ }^{\circ} \alpha_{\mu f}\left(\left\|x-x_{s}\right\|\right)=: \alpha_{L}\left(\left\|x-x_{s}\right\|\right)$ with the class- $K_{\infty}$ function $\alpha_{L}$. Due to (29), we have that

$$
J_{T}^{c l}\left(x_{0}\right) \leq J_{N}^{*}\left(x_{0}\right)+T L_{e}\left(x_{s}, u_{s}\right)+\sum_{k=0}^{T-1} \theta_{2}\left(\left\|x_{0}-x_{s}\right\|, k\right)
$$

where $\theta_{2}\left(\left\|x_{0}-x_{s}\right\|, k\right)=\alpha_{L^{\circ}} \gamma_{3}^{-1}\left(\rho_{2}\left(\left\|x_{0}-x_{s}\right\|\right) e^{-\bar{a} \beta k}\right)$ and it is a class- $K L$ function.

From the dynamic programming principle, $J_{N}^{*}(x)$ satisfies that

$$
J_{N}^{*}\left(x_{0}\right) \leq \inf _{\mathbf{u} \in U_{N}\left(x_{0}\right)}\left\{J_{T}\left(x_{0}, \mathbf{u}\right)+J_{N-T}^{*}\left(\varphi\left(T ; x_{0}, \mathbf{u}\right)\right)\right\} .
$$

Let $r=\rho_{2}^{-1}(c)$. For $T \in \mathbf{I}_{1: N-1}$ and $\mathbf{u}$ steering (1) to the ball $B_{r}$ within $T$ time steps, we obtain that

$$
\begin{aligned}
J_{T}\left(x_{0}, \mathbf{u}\right) & =J_{T}\left(x_{0}, \mathbf{u}\right)+J_{N-T}^{*}\left(\varphi\left(T ; x_{0}, \mathbf{u}\right)\right)-J_{N-T}^{*}\left(\varphi\left(T ; x_{0}, \mathbf{u}\right)\right) \\
& \geq J_{N}^{*}\left(x_{0}\right)-(N-T)\left[\alpha_{L}(r)+L_{e}\left(x_{s}, u_{s}\right)\right]
\end{aligned}
$$

Note that for $T \geq N$, the non-negativity of $L_{e}(x, u)$ implies that the inequality $J_{T}(x, \mathbf{u}) \geq J_{N}{ }^{*}(x)$ holds for all admissible $\mathbf{u} \in U_{T}(x)$, implying (34) again. Substituting (34) to (33) leads to 


$$
J_{T}^{c l}\left(x_{0}\right) \leq J_{T}\left(x_{0}, \mathbf{u}\right)+(N-T) \alpha_{L}(r)+N L_{e}\left(x_{s}, u_{s}\right)+\sum_{k=0}^{T-1} \theta_{2}\left(\left\|x_{0}-x_{s}\right\|, k\right)
$$

for all admissible $\mathbf{u} \in U_{T}(x)$ steering (1) to $B_{r}$ within $T$ time steps, which is equal to (30).

It is remarked that from Proposition 1 the transient performance given here does not admit transient optimality in the sense of [11] because the error term will not vanish as $N \rightarrow \infty$ and $\left\|\varphi\left(T ; x_{0}, \mu_{N}(\cdot)\right)-x_{s}\right\| \rightarrow 0$. Nevertheless, the closed-loop system (17) admits the asymptotic time average performance.

Proposition 2: Under the assumptions in Theorem 1, the closed-loop system (17) admits the asymptotic time average performance, i.e.,

with the region of attraction $X_{N}$.

$$
\lim _{K \rightarrow \infty} \inf J_{K}^{c l}(x) / K \leq L_{e}\left(x_{s}, u_{s}\right)
$$

Proof: It is straightforward to test that the suboptimal controller defined by the sequence (18) can asymptotically stabilize the system (1) to $x_{s}$ with $X_{N}$. Then following the proof of Theorem 7 in [13], one can show the inequality (35) and hence, the proof of this claim is omitted.

Note that since the asymptotic average performance may not be a singleton in the context of economic NMPC, a lower bound on the asymptotic average performance under the proposed EMPC scheme is considered in (36) from $[4,8,13]$. In addition, the average transient performance over a finite time window is not guaranteed to be better than $L_{e}\left(x_{s}, u_{s}\right)$ and it may take any value. Recently [11] established optimal transient performance estimates for the EMPC without terminal constraint.

Due to the constraint (7f), the contractive factor $\beta$ will affect the predicted optimal performance $J_{N}{ }^{*}(x)$. For clarity, let $x=x_{k}, J_{N}^{*}(x, \beta):=J_{N}^{*}(x), \eta(\beta):=\eta$ and $U_{N}(x, \beta):=U_{N}(x, \eta)$ for a selected $0<\beta \leq 1$. From Theorem 1 , the feasible set $U_{N}(x, \beta)$ is always non-empty for any $x \in X_{N}$.

Proposition 3: Under the assumptions in Theorem 1, the predicted optimal performance satisfies that $J_{N}{ }^{*}\left(x, \beta_{1}\right) \leq$ $J_{N}^{*}\left(x, \beta_{2}\right)$ for any $0<\beta_{1}<\beta_{2} \leq 1$ and $x \in X_{N}$.

Proof: The proof consists of two parts. We first prove that $U_{N}\left(x, \beta_{2}\right) \subseteq U_{N}\left(x, \beta_{1}\right)$ for $0<\beta_{1}<\beta_{2} \leq 1$ and any $x \in X_{N}$, and then prove the proposition by contradiction under the assumptions.

Part 1. From the proof of Lemma 1, it is known that $\eta(\beta)>0$ for any $0<\beta \leq 1$. For two values $0<\beta_{1}<\beta_{2} \leq 1$, then we have $\eta\left(\beta_{2}\right)<\eta\left(\beta_{1}\right)$ due to the inequality (22). Consider the sets $U_{N}\left(x, \beta_{1}\right)$ and $U_{N}\left(x, \beta_{2}\right)$, and pick any $\mathbf{u}\left(\beta_{2}\right) \in U_{N}\left(x, \beta_{2}\right)$. We obtain that $J_{a}\left(x, \mathbf{u}\left(\beta_{2}\right)\right) \leq \eta\left(\beta_{2}\right)<\eta\left(\beta_{1}\right)$, which leads to $\mathbf{u}\left(\beta_{2}\right) \in U_{N}\left(x, \beta_{1}\right)$. Due to arbitrariness of $\mathbf{u}\left(\beta_{2}\right) \in U_{N}\left(x, \beta_{2}\right)$, it is obtained that $U_{N}\left(x, \beta_{2}\right) \subseteq U_{N}\left(x, \beta_{1}\right)$ for $0<\beta_{1}<\beta_{2} \leq 1$ and any $x \in X_{N}$.

Part 2. By contradiction, it is assumed that $J_{N}{ }^{*}\left(x, \beta_{1}\right)>J_{N}{ }^{*}\left(x, \beta_{2}\right)$ for any $0<\beta_{1}<\beta_{2} \leq 1$ and $x \in X_{N}$. Let $\mathbf{u}^{*}\left(\beta_{i}\right) \in U_{N}\left(x, \beta_{i}\right)$ be the optimal solution corresponding with $J_{N}^{*}\left(x, \beta_{i}\right)$ for $i=1,2$. From Part 1 , we have $\mathbf{u}^{*}\left(\beta_{2}\right) \in U_{N}\left(x, \beta_{1}\right)$, which implies that there exists a solution $\mathbf{u}^{*}\left(\beta_{2}\right)$ such that $J_{N}^{*}\left(x, \beta_{2}\right)<J_{N}^{*}\left(x, \beta_{1}\right)$ in $U_{N}\left(x, \beta_{1}\right)$. This contradicts the optimality of $J_{N}^{*}\left(x, \beta_{1}\right)$ in $U_{N}\left(x, \beta_{1}\right)$. Hence, this proposition holds.

Proposition 3 shows that the predicted optimal performance $J_{N}{ }^{*}(x)$ will be degraded when increasing the value of $\beta$, which, on the other hand, speeds up the decaying of $V(x)$ and hence improves stability of the closed-loop system. In this sense, the loss of optimality of $J_{N}^{*}(x)$ can be regarded as a price that one has to pay for ensuring feasibility and stability of the EMPC controller. This mechanism is the so-called Stability Price in [15].

Remark 5: It should be emphasized that $J_{N}{ }^{*}(x)$ is a measure of open-loop performance and generally $J_{N}{ }^{*}(x) \neq J_{N}^{c l}(x)$ due to the receding horizon nature of MPC. Hence, Proposition 3 and Stability Price may not hold for $J_{N}^{c l}(x)$. Given that two different values of $\beta$ will generate different closed-loop trajectories, even if they start from the same initial state $x_{0}$, it is a great challenge to theoretically establish analogues of both Proposition 3 and Stability Price for $J_{N}^{c l}(x)$. Nevertheless, in practice Stability Price can provide guidance to tune the contractive factor $\beta$ and this will be illustrated by a numerical example.

\section{NUMERICAL EXAMPLE}

To illustrate the performance of the proposed EMPC scheme, we consider the isothermal chemical reactor system [5]

$$
\begin{aligned}
\frac{d c_{\mathrm{a}}}{d t} & =\frac{Q_{\mathrm{in}}}{V_{\mathrm{R}}}\left(c_{\text {ain }}-c_{\mathrm{a}}\right)-\sigma c_{\mathrm{a}}^{2} \\
\frac{d c_{\mathrm{b}}}{d t} & =\frac{Q_{\text {in }}}{V_{\mathrm{R}}}\left(c_{\mathrm{bin}}-c_{\mathrm{b}}\right)+\sigma c_{\mathrm{a}}^{2}
\end{aligned}
$$


where $c_{\mathrm{a}}$ and $c_{\mathrm{b}}$ are the molar concentrations of species 'a' and ' $\mathrm{b}$ ', respectively, $c_{\text {ain }}$ and $c_{\mathrm{bin}}$ are the feed concentrations of ' $\mathrm{a}$ ' and ' $\mathrm{b}$ ', and $Q_{\text {in }}$ is the flow through the reactor. The volume of the reactor $V_{\mathrm{R}}=10 \mathrm{~L}$ and the rate constant $\sigma=1.2 \mathrm{~L} /(\mathrm{mol} \cdot \mathrm{min})$. In this study, the concentrations $c_{\mathrm{a}}$ and $c_{\mathrm{b}}$ are the states $x_{1}$ and $x_{2}$, respectively, and the flow $Q_{\text {in }}$ is the control $u$. The constraints are imposed on the state and control variables by the form of $x_{i} \in[0,1]$ for $i=1,2$ and $u \in[0,15]$. The process economics are defined as the price of product $\mathrm{b}$ and a separation cost

$$
L_{e}(x, u)=0.5 u-2 x_{2} u \text {. }
$$

The optimal steady-state point for this cost is computed as $\left(x_{s}, u_{s}\right)=(0.5785,0.4215,9.5258)$. In order to design the contractive constraint $(7 \mathrm{f})$, we define the following positive definite function

$$
L_{a}(x, u)=\left\|x-x_{s}\right\|_{2}^{2}+\left\|u-u_{s}\right\|_{2}^{2} .
$$

Let the system $(A, B)$ be the linearized model of (36) at $\left(x_{s}, u_{s}\right)$. From the LQ optimal control approach, solving the Riccati equation $P A+A^{\mathrm{T}} P-P B B^{\mathrm{T}} P+I=0$ leads to a positive definite matrix solution $P=\left[\begin{array}{ll}0.3448 & 0.2213 \\ 0.2213 & 0.5248\end{array}\right]$. Then we construct the triplet as $E_{a}(x)=\left(x-x_{s}\right)^{\mathrm{T}} P\left(x-x_{s}\right), X_{f}=\left\{x \in R^{2}: E_{a}(x) \leq 0.0613\right\}$ and $\mu_{f}(x)=\left[\begin{array}{ll}-0.0052 & 0.0128\end{array}\right]\left(x-x_{s}\right)+u_{s}$, which satisfies Assumption 1 of Section III. Assumption 2 is also satisfied given the function forms of $f$ and $L_{e}$. Since it is hard to directly validate Assumption 3 during numerical optimization, here we imposed the condition $\left\|u_{i \mid k}(x)-u_{s}\right\| \leq \sigma\left\|x-x_{s}\right\|$ with $\sigma=2000$ for $i \in \boldsymbol{I}_{0: N-1}$ and $x \in X_{f}$ on the optimization operation to fulfill Assumption 3.

The system (36) is discretized with a sampling time $0.1 \mathrm{~min}$ and the Euler's first-order approximation is employed for all derivatives. Let the prediction horizon steps $N=4$ and the simulation time steps be 70 . The solution at time $k$ was used as an initial guess for solving the optimization problems at $k+1$. Moreover, all optimization problems had been solved by the fmincon function with the SQP algorithm in MATLAB V7.1 on the computer of MS WINDOWS 7.0 Enterprise and an Intel® Core i5 CPU with 2.3 GHz and 4 GB RAM.
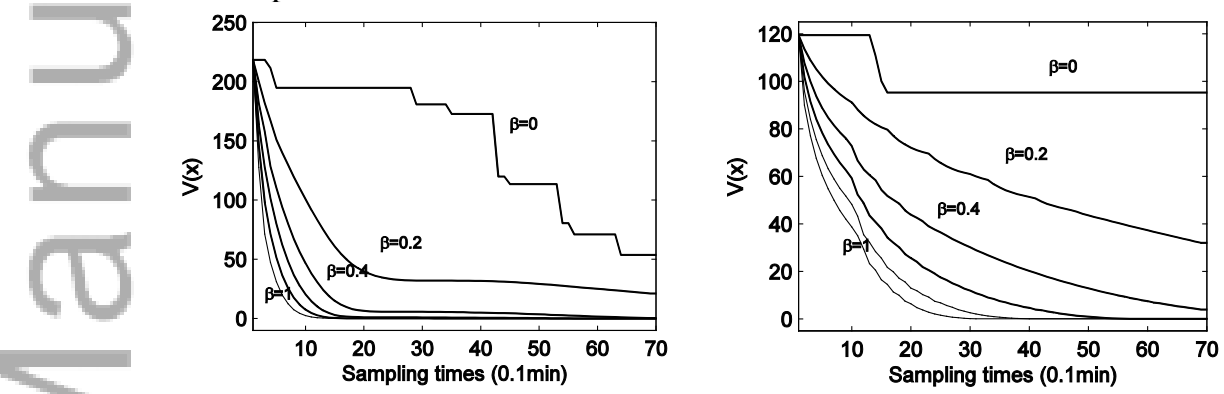

Fig. 1 Time profiles of $V(x)$ for different values of $\beta$. Left plot: initial state (I); right plot: initial state (II).
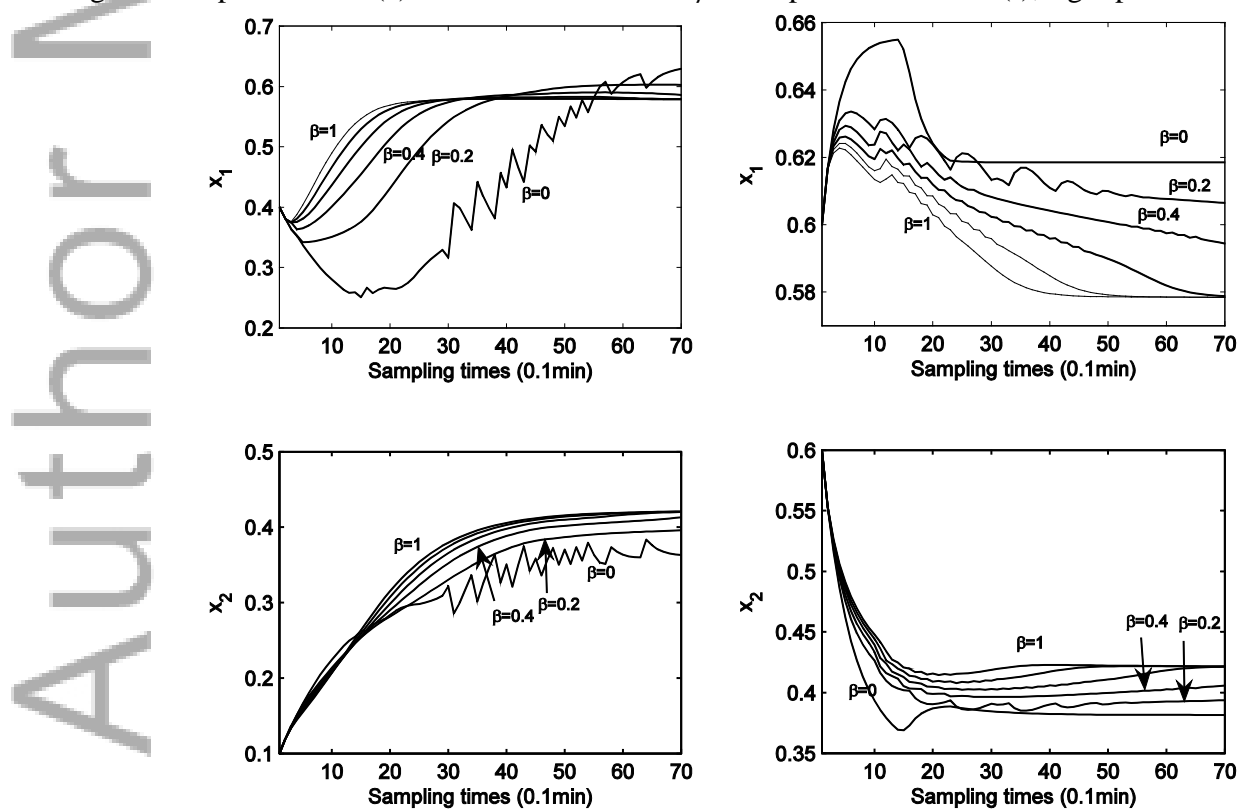

Fig. 2 Closed-loop state trajectories for different values of $\beta$. Left plot: initial state (I); right plot: initial state (II). 
We pick two initial states (I) $(0.4,0.1)$ and (II) $(0.6,0.6)$. Figs. $1-2$ separately show the time profiles of $V(x)$ and the state trajectories for different values of the contractive factor $\beta$, where the left plot is associated with the initial state (I) and the right the initial state (II). The profiles depicted in both figures are for $\beta$ in the interval $[0,1]$ with increments of 0.2 . It can be seen from both figures that 1 ) for all $0<\beta \leq 1$, the value functions are strictly decreasing and thus stability of the closed-loop system is established; 2) for larger values of $\beta$, the value functions decay more quickly, which implies that the closed-loop system approaches to the steady-state point $\left(x_{s}, u_{s}\right)$ more quickly. It should be emphasized that the condition of $0<\beta \leq 1$ is sufficient but not necessary to guarantee stability of the closed-loop system, which can be illustrated by the profile of $V(x)$ corresponding to $\beta=0$ in the left subfigure.

We define a transient time window $[0, T]$ of (36) such that the closed-loop state $\varphi\left(T ; x_{0}, \mu_{N}(\cdot)\right)$ enters the range of $\pm 5 \%$ the steady state $x_{s}$. Denote by $J_{T}^{c l}(\mathrm{I})$ and $J_{T}^{c l}(\mathrm{II})$ the transient economic performances obtained by applying Algorithm 1, starting from the initial states (I) and (II), respectively. Table 1 presents these values for the different values of $\beta$. As can be seen, the transient performance obtained by the proposed scheme is a decreasing function on $\beta$. That is, the transient performance will be improved by reducing the value of $\beta$. This observation may be explained to some extent by the Stability Price of the predicted performance since the constraint ( $7 \mathrm{f}$ ) is relaxed by reducing $\beta$ and hence, the predicted performance can be improved. However, it is seen from Fig. 2 that the convergence speed of the closed-loop system gets slower as $\beta$ becomes smaller. In this sense, we can select the value of $\beta$ to make a tradeoff between the economic performance and stability of the closed-loop system.

Table 1. The transient economic performances for different values of $\beta$ under $N=4$

\begin{tabular}{ccccccc}
\hline$\beta$ & 0.2 & 0.4 & 0.6 & 0.8 & 1.0 \\
\hline$J_{T}^{c l}(\mathrm{I})$ & -209.8552 & -60.0956 & -45.4511 & -38.4738 & -36.7232 \\
$J_{T}^{c l}(\mathrm{II})$ & -417.1054 & -205.7893 & -114.7035 & -104.2869 & -93.4900 \\
\hline
\end{tabular}

Table 2. The transient economic performances and computational times for different values of $N$ under $\beta=0.8$

\begin{tabular}{ccccccc}
\hline & \multicolumn{1}{c}{4} & 5 & 10 & 15 & 20 \\
\cline { 2 - 7 } & $J_{T}^{c l}(\mathrm{I})$ & -38.4738 & -42.0817 & -40.3958 & -38.3085 & -32.0736 \\
$J_{T}^{c l}(\mathrm{II})$ & -104.2869 & -85.6629 & -81.8655 & -99.9914 & -107.4492 \\
\hline$T_{\max }(\mathrm{ms})$ & 446.1 & 554.4 & 735.4 & 957.7 & 2209 \\
$T_{\text {ava }}(\mathrm{ms})$ & 29.5 & 31.2 & 51.9 & 86.3 & 170.1 \\
\hline
\end{tabular}

Table 2 tabulates the transient economic performances and computational times by applying Algorithm 1 for different lengths of the prediction horizon $N$ under the fixed $\beta=0.8$. It is observed that the obtained transient performance can not be improved by only lengthening or reducing the prediction horizon $N$. In other words, the obtained transient performance is not a monotone function on $N$. However, the computational times for calculating control actions, over all sampling times of all simulations, are reduced when we select a shorter $N$.

In what follows, we consider the initial state (II) and assess the three controllers applied by the single-layer contractive EMPC proposed here (SC-EMPC), the double-layer contractive EMPC in [15] (DC-EMPC) and the single-layer dissipative EMPC in [6] (SD-EMPC). Note that the system (37) is not dissipative w.r.t the economic criterion (38). Hence the following regularized function followed from [6] is used for SD-EMPC:

$$
L_{e}(x, u)=0.5 u-2 x_{2} u+0.1\left(u-u_{s}\right)^{2}
$$

with a penalty function $V_{f}(x)=E_{a}(x)+\left[\begin{array}{lll}-4.0541 & -9.7533\end{array}\right]\left(x-x_{s}\right)$ and the terminal region $X_{f}$. Moreover, in order to guarantee initial feasibility of DC-EMPC, we select the prediction horizon $N=15$ for all three controllers. It is noted that initial feasibility of DC-EMPC is ensured only if $N \geq 10$ but initial feasibility of SC-EMPC and SD-EMPC is ensured for $N=4$ due to the terminal inequality constraint.

\begin{tabular}{l} 
Table 3. The transient economic performances and computational times for three controllers \\
\cline { 2 - 5 } \\
\begin{tabular}{ccccc} 
SC-EMPC & DC-EMPC & SD-EMPC & SC-EMPC \\
& $(15,0.8)$ & $(15,0.2)$ & $(15,--)$ & $(4,0.6)$ \\
\hline$J_{T}^{c l}(\mathrm{II})$ & -99.9914 & -109.5727 & -109.6129 & -114.7035 \\
$T_{\max }(\mathrm{ms})$ & 957.7 & 1254.9 & 498.8 & 508.2
\end{tabular}
\end{tabular}




\begin{tabular}{lllll}
$T_{\text {ava }}(\mathrm{ms})$ & 86.3 & 186.1 & 40.6 & 32.1 \\
\hline
\end{tabular}

Table 3 gives the transient economic performances and computational times obtained by separately applying the three controllers, respectively, where $\beta=0.8$ for SC-EMPC and $a=0.2$ for DC-EMPC. From the $1^{\text {st }}$ to $3^{\text {rd }}$ column of Table 3, it is known that the transient economic performance of DC-EMPC is almost equal to that of SD-EMPC, and both DC-EMPC and SD-EMPC have better transient performances than SC-EMPC. This implies that the transient performance of SC-EMPC admits no transient optimality in the sense of [11]. However, we known from Tables 1 and 2 that the transient economic performance of SC-EMPC has no monotonicity w.r.t $N$ but it is a decreasing function on $\beta$. Furthermore, it is observed from the three columns that there is significant difference in the computational time among three controllers. We can select a shorter $N$ and a smaller $\beta$, e.g. $(N, \beta)=(4,0.6)$, to improve the transient economic performance of SC-EMPC as well as reducing its computational times, as shown in the last column of this table.

Figs. 3 and 4 show the time evolutions of the closed-loop states and control inputs by separately applying the three controllers, where $(N, \beta)=(4,0.6)$ for SC-EMPC. As expected, the three closed-loop systems are asymptotically stable at $x_{s}$ in the presence of the state and control constraints, but they approach the economic setpoint in different ways. In particular, comparing the solid lines to the dashed and dotted lines in figs. 3 and 4, it is that both DC-EMPC and SD-EMPC produce smoother closed-loop state responses and control signals than SC-EMPC. Note that one can adjust $\beta$ to mitigate the oscillatory behavior resulted from SC-EMPC (see Fig.2).
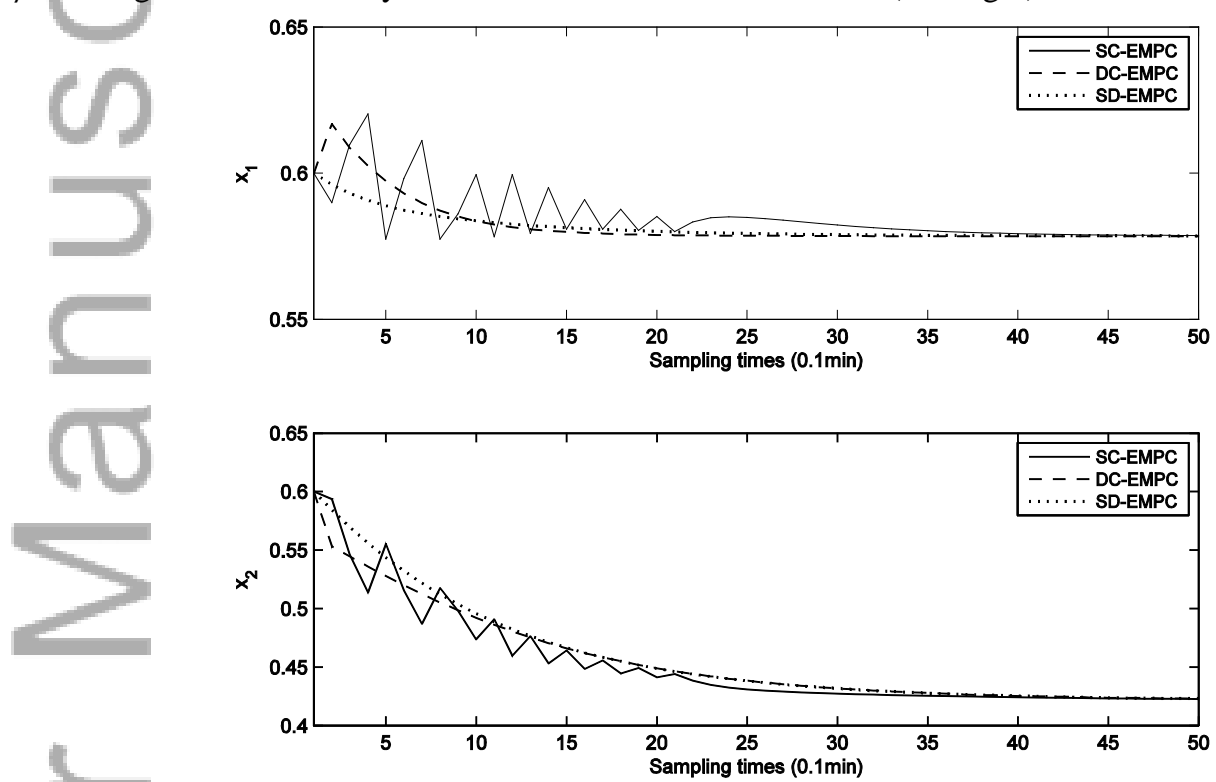

Fig. 3 Time evolutions of the closed-loop states starting from the initial state II, associated with three controllers.

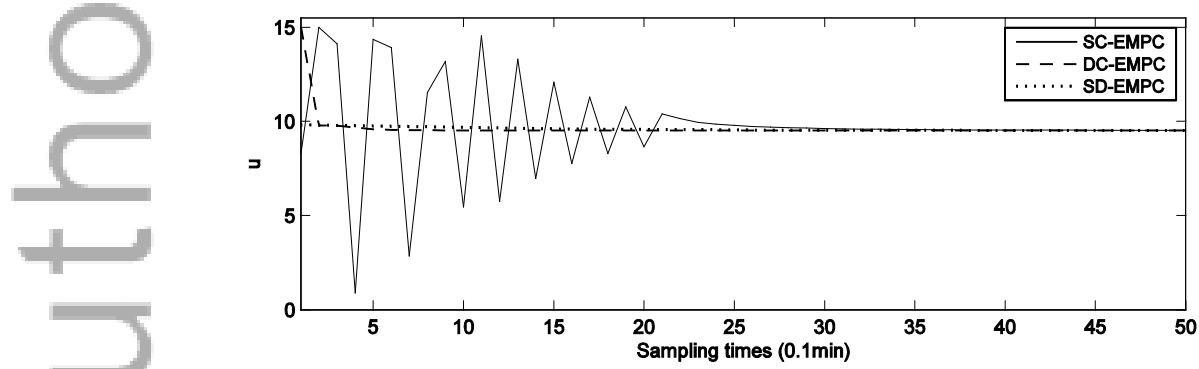

Fig. 4 Time profiles of the inputs applied by three controllers, associated with the initial state II.

\section{CONCLUSIONS}

This paper proposed a contractive EMPC scheme with guaranteed asymptotic stability for constrained nonlinear systems. By imposing a special contractive constraint, based on the terminal region and terminal penalty, into the EMPC optimization problem, we derived the sufficient conditions for guaranteeing recursive feasibility and 
stability of EMPC. Moreover, we analyzed the transient economic performance and established the relation of the predicted performance to the contractive factor. The example of an isothermal chemical reactor demonstrated the effectiveness of the EMPC scheme proposed here.

\section{REFERENCES}

[1] Ellis M, Durand H, Christofides PD. A tutorial review of economic model predictive control methods. Journal of Process Control, 2014, 24(8), 1156-1178.

[2] Tran T, Ling KV, Maciejowski JM. Economic model predictive control-A review. In proceedings of the $31^{\text {st }}$ Int. Symposium on Automation and Robotics in Construction and Mining, University of Technology Sydney, 2014: 35-42.

[3] Mayne DQ. Model predictive control: Recent developments and future promise. Automatica, 2014, 50, 2967-86.

[4] Angeli D, Amrit R, Rawlings JB. On average performance and stability of economic model predictive control. IEEE Transactions on Automatic Control, 2012, 57(7): 1615-1626.

[5] Diehl M, Amrit R, Rawlings J B. A Lyapunov function for economic optimizing model predictive control. IEEE Transactions on Automatic Control, 2011, 56(3), 703-707.

[6] Amrit R, Rawlings JB, Angeli D. Economic optimization using model predictive control with a terminal cost. Annual Reviews in Control, 2011, 35(2), 178-186.

[7] Müller MA, Angeli D, Allgöwer F, et al. Convergence in economic model predictive control with average constraints. Automatica, 2014, 50(12): 3100-3111.

[8] Rawlings JB, Angeli D, Bates CN. Fundamentals of economic model predictive control. In proceedings of the $51^{\text {st }}$ IEEE Conference on Decision and Control, Maui, Hawaii, 2012, pp. 3851-3861.

[9] Fagiano L, Teel AR. Generalized terminal state constraint for model predictive control. Automatica, 2013, 49(9): 2622-2631.

[10] Grüne L. Economic receding horizon control without terminal constraints. Automatica, 2013, 49(3), 725-734.

[11] Grüne L, Stieler M. Asymptotic stability and transient optimality of economic MPC without terminal conditions. Journal of Process Control, 2014, 24, 1187-1196.

[12] Heidarinejad M, Liu J, Christofides PD. Economic model predictive control of nonlinear process systems using Lyapunov techniques, AIChE J., 2012, 58, 855-870.

[13] Ellis M, Christofides PD. On finite-time and infinite-time cost improvement of economic model predictive control for nonlinear systems. Automatica, 2014, 50(10), 2561-2569.

[14] He D, Wang L, Sun J. On stability of multiobjective NMPC with objective prioritization. Automatica, 2015, 57, 189-198.

[15] Zavala VM. A multiobjective optimization perspective on the stability of economic MPC. http://www.mcs.anl.gov/papers/ P5283-0115.pdf, 2015.

[16] Pannocchia G, Rawlings JB, Wright SJ. Conditions under which suboptimal nonlinear MPC is inherently robust. System \& Control Letters, 2011, 60, 747-755.

[17] Cannon M. Efficient nonlinear model predictive control algorithms. Annual Review in Control, 2004, 28, $229-237$.

[18] He DF, Yu SM, Song XL. Lyapunov-based dual-mode method for economic optimization model predictive control. In Pro. $53^{\text {rd }}$ IEEE CDC, Los Angeles, CA, USA, 2014, 3505-3510.

[19] Yang TH, Polak E. Moving horizon control of nonlinear systems with input saturation, disturbances and plant uncertainty. Int. J. Control, 1993, 58(4), 875-903.

[20] Kothare SLO, Morari M. Contractive model predictive control for constrained nonlinear systems. IEEE Transactions on Automatic Control, 2000, 45(6), 1053-1071.

[21] Chen H, Allgöwer F. A quasi-infinite horizon nonlinear model predictive control scheme with guaranteed stability. Automatica, 1998, 34(10), 1205-1217.

[22] Fontes FACC. A general framework to design stabilizing nonlinear model predictive controllers. Systems \& Control Letters, 2001, 42, 127-143.

[23] Magni L, De Nicolao G, Magnani L, Scattolini R. A stabilizing model-based predictive control algorithm for nonlinear systems. Automatica, 2001, 37, 1351-1362.

[24] Limon D, Bravo JM, Alamo T, Camacho EF. Robust MPC of constrained nonlinear systems based on interval arithmetic. IEE Proceedings on Control Theory Applications, 2005, 152(3), 325- 332.

[25] Mhaskar P, El-Farra NH, Christofides PD. Stabilization of nonlinear systems with state and control constraints using Lyapunov-based predictive control. System \& Control Letters, 2006, 55, 650-659.

[26] Maree JP, Imsland L. Performance and stability for combined economic and regulatory control in MPC. In IFAC World Congress, Cape Town, South Africa, 2014, V.9, 639-645. 
Table 1. The transient economic performances for different values of $\beta$ under $N=4$

\begin{tabular}{cccccc}
\hline$\beta$ & 0.2 & 0.4 & 0.6 & 0.8 & 1.0 \\
\hline$J_{T}^{c l}(\mathrm{I})$ & -209.8552 & -60.0956 & -45.4511 & -38.4738 & -36.7232 \\
$J_{T}^{c l}(\mathrm{II})$ & -417.1054 & -205.7893 & -114.7035 & -104.2869 & -93.4900 \\
\hline
\end{tabular}

Table 2. The transient economic performances and computational times for different values of $N$ under $\beta=0.8$

\begin{tabular}{ccccccc}
$N$ & 4 & 5 & 10 & 15 & 20 \\
\cline { 2 - 7 } & $J_{T}^{c l}(\mathrm{I})$ & -38.4738 & -42.0817 & -40.3958 & -38.3085 & -32.0736 \\
$J_{T}^{c l}(\mathrm{II})$ & -104.2869 & -85.6629 & -81.8655 & -99.9914 & -107.4492 \\
\cline { 2 - 7 } & $T_{\max }(\mathrm{ms})$ & 446.1 & 554.4 & 735.4 & 957.7 & 2209 \\
& $T_{\text {ava }}(\mathrm{ms})$ & 29.5 & 31.2 & 51.9 & 86.3 & 170.1 \\
\hline
\end{tabular}

Table 3. The transient economic performances and computational times for three controllers

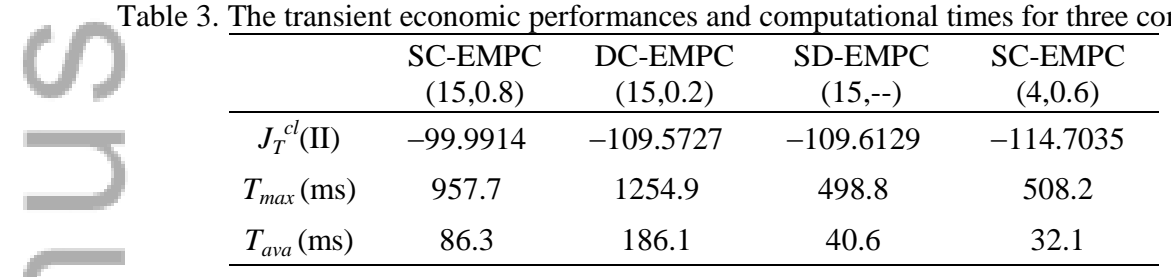

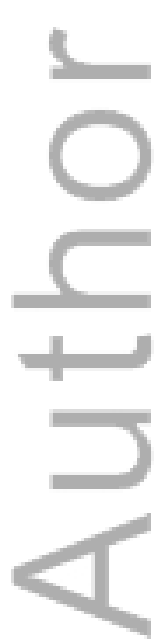

